\title{
Campylobacter 0:41 isolation in Guillain-Barré syndrome
}

\author{
E A Goddard, A J Lastovica, A C Argent
}

\begin{abstract}
Over a period of 15 months, 17 children were admitted to the Red Cross War Memorial Children's Hospital (RCWMCH) in Cape Town with Guillain-Barré syndrome. Stool specimens were collected from 14 children and campylobacter was isolated from nine. Six of the nine isolates of Campylobacter jejuni were further identified as $C$ jejuni biotype 2 , serotype $0: 41$. This biotype 2 , serotype $0: 41$ has been identified in only 12 of the 7119 campylobacter isolates at the RCWMCH over a 19 year period. Eight of the nine patients with campylobacter isolates and one of five with negative stool cultures required ventilation. Patients with $C$ jejuni biotype 2 , serotype $0: 41$ were ventilated for a mean (SD) of 33.5 (19.4) days, whereas patients with other campylobacter isolates were ventilated for 17.3 (3.8) days. This is the first report of campylobacters of serotype $0: 41$ in Guillain-Barré syndrome and could reflect a geographical isolation of this strain.
\end{abstract}

(Arch Dis Child 1997;76:526-528)

Keywords: Guillain-Barré syndrome; Campylobacter jejuni

The Guillain-Barré syndrome (GBS) is the most common cause of acute neuromuscular paralysis. Although GBS has been associated with a wide variety of preceding infections, campylobacter infections appear to be the most common preceding event, with evidence of infection in up to $38 \%$ of adults with GBS. ${ }^{12}$ There have been a few reports of an association of childhood GBS and campylobacter infection. $^{3-5}$

At the Red Cross War Memorial Children's Hospital (RCWMCH) in Cape Town, an average of 10 cases of GBS have been seen each year since 1975. During the period June 1994 to August 1995 a total of 23 children with GBS were seen at the hospital, of whom 17 were admitted to the intensive care unit. This paper reports the associations between campylobacter infection and GBS in these patients.

\section{Patients and methods}

Stool specimens were collected from patients admitted to the intensive care unit at RCW$\mathrm{MCH}$ between June 1994 and August 1995 with clinical features of GBS. Patients were included in the study if they fulfilled the diagnostic criteria for acute GBS as published by Asbury and Cornblath. ${ }^{6}$ Clinical data were obtained from a retrospective analysis of the patients' charts.

ISOLATION AND GROWTH

All stool specimens were cultured by established methods. Gram negative motile, spiral, or curved rods were cultured on tryptose blood agar plates (CM233, Oxoid Ltd) containing $10 \% \mathrm{v} / \mathrm{v}$ unlysed horse blood. Culture plates were incubated at $37^{\circ} \mathrm{C}$ under microaerophilic (12\% carbon dioxide, $88 \%$ air, and $95 \%$ humidity) growth conditions.

\section{BIOTYPING AND SEROTYPING}

Identification was accomplished by established tests and all isolates were biotyped by the scheme of Skirrow and Benjamin. ${ }^{7}$ Serotyping on the basis of thermostable somatic (O) lipopolysaccharide antigens was performed with the 66 antisera of the Penner scheme. ${ }^{8}$ Isolates were also serotyped against an additional 30 antisera to new serotypes not included in this scheme.

\section{Results}

Over a period of 15 months, 17 children aged 14-108 (median 47) months were admitted to the intensive care unit of the RCWMCH in Cape Town with GBS (table 1).

Campylobacter species were isolated from nine of the 14 patients from whom stool specimens were obtained. Six of these isolates were identified as $C$ jejuni biotype 2, serotype 0:41. The other three campylobacter isolates were $C$ concisus, $C$ upsaliensis, and $C$ jejuni biotype 1 .

Before presentation with GBS only three of the nine patients with a positive campylobacter culture had a history of a preceding diarrhoeal illness, four had had a preceding upper respiratory tract infection, and two had no prior infection.

The GBS in patients with campylobacter isolates was more severe than in patients with negative stool cultures. Eight of the nine patients with campylobacter isolates and one of five patients with negative stool cultures required ventilation $(\mathrm{p}<0.05)$ with a mean duration of ventilation of 28.1 days. The duration of admission to hospital was also prolonged in patients with campylobacter (mean $43 v 9.8$ days, $\mathrm{p}<0.01$ ). Only one patient who was ventilated for more than 20 days had a negative stool culture for campylobacter isolates. One patient from whom no stool was collected was ventilated for more than 20 days.

Among patients with campylobacter isolates, those with $C$ jejuni biotype 2, serotype 0:41 had more severe disease. Overall, patients with $C$ jejuni biotype 2, serotype 0:41 were ventilated 
Table1 Patient details

\begin{tabular}{lllll}
\hline Patient No & Age (months) & Stool culture & $\begin{array}{l}\text { Duration of ventilation } \\
\text { (days) }\end{array}$ & $\begin{array}{l}\text { Duration of stay in hospital } \\
\text { (days) }\end{array}$ \\
\hline 1 & 16 & None cultured & 24 & 34 \\
2 & 73 & None cultured & 0 & 3 \\
3 & 45 & None cultured & 0 & 6 \\
4 & 108 & None cultured & 0 & 3 \\
5 & 48 & None cultured & 0 & $11.8(13.0)$ \\
Mean (SD) & $58.0(34.5)$ & Biotype 2, serotype 0:41 & $4.8(10.7)$ & 51 \\
6 & 22 & Biotype 2, serotype $0: 41$ & 37 & 42 \\
7 & 108 & Biotype 2, serotype $0: 41$ & 57 & 74 \\
8 & 84 & Biotype 2, serotype $0: 41$ & 0 & 28 \\
9 & 36 & Biotype 2, serotype $0: 41$ & 29 & 51 \\
10 & 26 & Biotype 2, serotype 0:41 & 31 & $50.8(15.5)$ \\
11 & 22 & & $33.5(19.4)$ & 26 \\
Mean (SD) & $49.7(37.0)$ & C concisus & 19 & 23 \\
12 & 48 & C upsaliensis & 13 & 33 \\
13 & 47 & Biotype 1 & 20 & $27.3(5.13)$ \\
14 & 14 & & $17.3(3.8)$ & 13 \\
Mean (SD) & $20.3(37.0)$ & Not done & 0 & 71 \\
15 & 47 & Not done & 71 & 15 \\
16 & 64 & Not done & 8 & $33.0(33.0)$ \\
17 & 96 & & $26.3(38.9)$ & \\
Mean (SD) & $69(24.9)$ & & & \\
\hline
\end{tabular}

for 33.5 (95\% confidence interval (CI) 13.1 to 54.0) days, whereas patients with other campylobacter isolates were ventilated for 17.3 (95\% CI 7.9 to 26.7) days. The length of stay in hospital was also prolonged for patients with $C$ jejuni biotype 2, serotype 0:41 at 50.8 days (95\% CI 34.6 to 67.1 ) $v 27.3$ days (95\% CI 14.6 to 40.0 ). There was no difference in age between the groups of patients.

\section{Discussion}

The RCWMCH is one of two referral hospitals serving a population of three million people, $40 \%$ of whom are under the age of 14 years. ${ }^{9}$ GBS is said to occur at a rate of $0.6-1.1$ cases per 100000 children under 15 years of age each year, ${ }^{10}$ suggesting that 12 children should present each year. To have approximately 10 patients with severe disease admitted to the intensive care unit each year suggests that a severe form of the disease is seen in this area. The age of the children is also unusually low for this severity of disease. A retrospective multicentre review of paediatric patients with GBS described a bimodal distribution of age, with peaks at 4 and 12 years, and severe disease was found predominantly in older children. ${ }^{11}$ It is possible that children in the older group are seen at adult units and not referred to the paediatric hospital.

Our patients with campylobacter associated GBS (CAGBS) had a particularly severe form of GBS with a high requirement for (eight of nine) and duration of (mean 28.1 days) ventilation. By contrast, only one of 23 children with GBS reported by Briscoe et al ${ }^{12}$ required ventilation, as did $16 \%$ of patients reported by Korinthenberg and Schulte Monting. ${ }^{11}$ In a report of 11 paediatric patients with severe GBS, ${ }^{13}$ the nine patients who survived were ventilated for a median of 18 days, whereas the median duration of ventilation reported in a multicentre study was 10 days. ${ }^{11}$

Our patients with CAGBS were younger than those previously described, with the exception of the patients reported by De Bont et al (2 years) ${ }^{3}$ and Sugita et al (4 years). ${ }^{5}$ Patients reported by Kuroki et al were aged 7-14 years (median 11 years), ${ }^{4}$ whereas Yuki et al reported two patients aged 13 and 9 years with Miller-Fisher syndrome associated with campylobacter. ${ }^{14}$ The proportion of preceding gastrointestinal symptoms in our patients with CAGBS is similar to other studies of CAGBS where diarrhoea was noted in four of nine $e^{4}$ and 19 of 27 patients. ${ }^{15}$

Patients with CAGBS from whom $C$ jejuni biotype 2, serotype 0:41 was isolated had a particularly severe form of GBS requiring ventilation and admission to hospital for a mean of 33.5 and 50.8 days, respectively. It is possible that the two patients (one with no stool isolate of campylobacter and one with no stool culture) with severe disease and no evidence of campylobacter infection had had recent infection as the organisms are not always found in stools at the time of presentation. ${ }^{16}$ Of the seven patients with CAGBS (four of these had serotype 0:19) reported by Kuroki et al, ${ }^{4}$ none required ventilation. One patient with CAGBS ${ }^{3}$ required ventilation for six weeks, but this patient had the complicating issue of severe short bowel syndrome.

$C$ jejuni biotype 2, serotype 0:41 is an extremely rare biotype of campylobacter in this region. Only $35 C$ jejuni biotype 2 isolates have been identified from the 7119 campylobacter isolates characterised at the RCWMCH in the last 19 years. ${ }^{17}$ Of these 35 strains, 12 (including the GBS related isolates) were serotyped as $0: 41$. The serotype 0:41 isolates not associated with GBS were from six children with severe chronic diarrhoea. Previous reports of CAGBS have implicated bacterial strains of Penner serotype $0: 19^{18} 19$ and Lior biotype $11 .^{20}$ The incidence of serotype 0:19 in campylobacter stool isolates seen at RCWMCH is $<3 \%$ and GBS was not recognised in patients with this serotype.

Previous studies have suggested that there is an association between the development of GBS and the presence of antibodies to ganglioside $\mathrm{GM}_{\mathrm{M} 1}{ }^{5}{ }^{521-23}$ Several studies have suggested that there are similarities between the lipopolysaccharide structures of campylobacter and human gangliosides. ${ }^{24}{ }^{25}$ Of particular interest is evidence that lipopolysaccharides extracted from $C$ jejuni of serotype 0:19 and 0:4 
had core oligosaccharides with terminal structures resembling human gangliosides $G_{M 1}$ and $\mathrm{G}_{\mathrm{D} 1 \mathrm{a}}{ }^{26}$ To date, there is no published data about the lipopolysaccharide structures of $C$ jejuni biotype 2, serotype 0:41 and this should be studied further.

We did not perform nerve conduction studies in these patients and thus do not know whether our patients had patterns of acute inflammatory demyelinating polyneuropathy or acute motor axonal neuropathy. ${ }^{27}$

It is impractical to treat patients with $C$ jejuni positive stools with antibiotics to prevent the rare complication of GBS. Previous studies have suggested that treatment of patients with campylobacter associated GBS does not prevent the development of GBS. ${ }^{2328}$ It may be worth investigating the possibility that early treatment with erythromycin may shorten the duration of the illness, however. Although treatment with erythromycin does not affect the natural course of campylobacter enteritis, it does clear campylobacter from stools. In two studies of acute campylobacter enteritis erythromycin cleared campylobacter from the stools of all 28 patients within two days, and in a study of 10 convalescent excretors erythromycin treatment cleared the stool within 48 hours. $^{29}$ Thus erythromycin does clear the organism and if there is a cross reaction with molecular mimicry between campylobacter and the central nervous system, clearance of the organism and reduction of the antigen load may make a difference to the clinical course of the patients.

\section{Conclusions}

We have documented the association of a severe form of GBS in children with concurrent infection with campylobacter species and particularly with $C$ jejuni biotype 2, serotype $0: 41$ (an extremely rare serotype in this community). This highlights the importance of campylobacter infection as a possible cause of GBS and raises further possible directions for the treatment and investigation of pathogenetic mechanisms of GBS. We thank Mrs L Heuer for typing this paper. We also thank E le
Roux in the Department of Microbiology at the Red Cross War Roux in the Department of Microbiology at the Red Cross War
Memorial Children's Hospital for culturing of the stool Memorial Children's Hospital for culturing of the stool
specimens. This research was supported by grants from the specimens. This research was supported by grants from the
South African Medical Research Council and the University of Cape Town to AJL.

1 Kaldor J, Speed BR. Guillain-Barré syndrome and Campylobacter jejuni: serological study. BMF 1984;288:1867-70. 2 Rees JH, Gregson NA, Griffiths PL, et al. Campylobacter jejuni and Guillain-Barré syndrome. $Q \mathcal{F}$ Med 1993;86:62334 .

3 De Bont B, Matthews N, Abbott CK, et al. Guillain-Barré syndrome associated with campylobacter enteritis in a child. F Pediatr 1986;109:660-2.
4 Kuroki S, Haruta T, Yoshioka M, et al. Guillain-Barré syndrome associated with campylobacter infection. Pediatr Infect Dis f 1991;10:149-51.

5 Sugita K, Ishii M, Takanashi J, et al. Guillain-Barré syndrome associated with IgM anti-GM1 antibody following Campylobacter jejuni enteritis. Eur f Pediatr 1994;153: 181-3.

6 Asbury AK, Cornblath DR. Assessment of current diagnostic criteria for Guillain-Barré syndrome. Ann Neurol 1990; 27(suppl):S21-4.

7 Skirrow MB, Benjamin J. Differentiation of enteropathogenic campylobacter. F Clin Pathol 1980;33:1122.

8 Penner JL, Hennessy JN, Congi RV. Serotyping of Campylobacter jejuni and campylobacter cold on the basis of the

9 Bridgman DHM, Palmer I, Thomas WH, eds. South Africa's leading edge? A guide to the Western Cape economy. Cape Town: Wesgro, 1992

10 Ropper AH. The Guillain-Barré syndrome. $N$ Engl f Med 1992;329:1130-6.

11 Korinthenberg R, Schulte Monting J. Natural history and treatment effects in Guillain-Barré syndrome: a multicentre study. Arch Dis Child 1996;74:281-7.

12 Briscoe DM, McMenamin JB, O'Donohoe NV. Prognosis in Guillain-Barré syndrome. Arch Dis Child 1987;62:733-5.

13 Cole GF, Matthew DJ. Prognosis in severe Guillain-Barré syndrome. Arch Dis Child 1987;62:288-91.

14 Yuki N, Ichikawa H, Doi A. Fisher syndrome after Campylobacter jejuni enteritis: human leukocyte antigen and the bacterial serotype. $\mathcal{F}$ Pediatr 1995;126: 55-7.

15 Rees J, Soudain S, Gregson A, et al. Campylobacter jejuni infection and Guillain-Barré syndrome. $N$ Engl f Med 1995;333:1374-9.

16 Mishu B, Ilyas M, Koski CL, et al. Serologic evidence of previous Campylobacter jejuni infection in patients with the Guillain-Barré syndrome. Ann Intern Med 1993;118: 947-53.

17 Lastovica AJ, Goddard EA, Argent AC, et al. Guillain-Barré syndrome and Campylobacter jejuni serotype 0:41 infection [abst]. 96th General Meeting of the American Society for Microbiology, 1996: abst C-334.

18 Fujimoto S, Yuki N, Itoh T, et al. Specific serotype of Campylobacter jejuni associated with Guillain-Barré syndrome. F Infect Dis 1992;165:183.

19 Kuroki S, Saida T, Nukina M, et al. Campylobacter jejuni strains from patients with Guillain-Barré syndrome belong mostly to Penner serogroup 19 and contain $\beta-N$ acetylglucosamine residues. Ann Neurol 1993;33:243-7.

20 Enders U, Karch H. Toyka KV, et al. The spectrum of immune responses to Campylobacter jejuni and glycoconjugates in Guillain-Barré syndrome and in other neuroimmunological disorders. Ann Neurol 1993;34:136-44.

21 Vriesendorp FJ, Mishu B, Blaser MJ, et al. Serum antibodies to GMI, GDlb, peripheral nerve myelin, and Campylobacter jejuni in patients with Guillain-Barré syndrome and controls: correlation and prognosis. Ann Neurol 1993;34: 130-5.

22 Von Wulffen H, Hartard C, Scharein E. Seroreactivity to Campylobacter jejuni and gangliosides in patients with Gampylobacter jejuni and gangliosides in patients with-Barré syndrome. F Infect Dis 1994;1 170:823-33.

23 Ho TW, Mishu B, Li CY, et al. Guillain-Barré syndrome in northern China. Relationship to Campylobacter jejuni infection and anti-glycolipid antibodies. Brain 1995;118: 597-605.

24 Yuki N, Handa S, Taki T, et al. Cross-reactive antigen between nervous tissue and a bacterium elicits GuillainBarré syndrome: molecular mimicry between ganglioside GM1 and lipopolysaccharide from Penner's serotype 19 of Campylobacter jejuni. Biomedical Research 1992;13:451-3.

25 Yuki N, Taki T, Inagaki F, et al. A bacterium lipopolysaccharide that elicits Guillain-Barré syndrome has a GM1 ganglioside-like structure. $\mathcal{F}$ Exp Med 1993;178:1771-5.

26 Aspinall GO, Fujimoto S, McDonald AG, et al. Lipopolysaccharide from Campylobacter jejuni associated with Guillain-Barré syndrome patients mimic human gangliosides in structure. Infect Immun 1994;62:2122-5.

27 Griffin JW, Li CY, Ho TW, et al. Guillain-Barré syndrome in northern China. The spectrum of neuropathological northern China. The spectrum of neuropathological

28 Ropper AH. Campylobacter diarrhea and Guillain-Barré syndrome. Arch Neurol 1988;45:655-6.

29 Blaser MJ, Reller LB. Campylobacter enteritis. $N$ Engl $\mathcal{f}$ Med 1981;305:1444-51. 